\title{
Paraneoplastic cerebellar degeneration and limbic encephalitis in a patient with adenocarcinoma of the colon
}

\author{
T Tsukamoto, R Mochizuki, H Mochizuki, M Noguchi, H Kayama, M Hiwatashi, \\ T Yamamoto
}

\begin{abstract}
The rare association of two neurological paraneoplastic syndromes, paraneoplastic cerebellar degeneration and limbic encephalitis occurred in a 55 year old woman with a microscopic adenocarcinoma in a colonic polyp. Complete removal of the tumour by polypectomy brought about a favourable recovery from limbic encephalitis but the cerebellar ataxia remained. High titres of antineuronal nuclear antibody resembling anti-Hu were demonstrated in serum by immunohistochemistry using rat brain as a substrate. The antibody identified a protein band of $41 \mathrm{kDa}$ on a Western immunoblot.
\end{abstract}

$(\mathcal{F}$ Neurol Neurosurg Psychiatry 1993;56:713-716)

Paraneoplastic cerebellar degeneration ${ }^{1}$ and limbic encephalitis ${ }^{2}$ are two neurological paraneoplastic syndromes which rarely occur in association with various malignant tumours without direct neoplastic invasion into the nervous system. We report both these paraneoplastic syndromes in a patient with a minute adenocarcinoma in the submucosal tissue of a colonic polyp.

\section{Case report}

A 55 year old housewife became aware of dizziness in November 1987 and her gait became gradually unsteady. In July 1988 her difficulty in walking progressed rapidly, and she was no longer able to walk without support. CT brain scans showed no abnormality. On 22 August an ill-defined low density area was noted in the medial part of the right temporal lobe. It became larger and by 31 August extended to the base of the right frontal lobe. There were enhanced areas in the low density area. T2 weighted MRIs on 2 September also showed an area of homogeneous high intensity signals with a relatively distinct margin in the medial half of the right temporal lobe and the base of the frontal lobe. The medial part of the left temporal lobe showed slightly increased signals (fig 1). An EEG showed a low voltage delta wave focus in the right temporal lobe. A brain biopsy disclosed mild gliosis with perivascular lymphocytic infiltration and oedema. In November she was emaciated, having lost $17 \mathrm{~kg}$ of weight in one year. She was alert, but exhibited scanning speech and severe disequilibrium. Mild impairment of memory function and attention were noted. Tendon reflexes were slightly hyperactive throughout, but without a Babinski sign. The sensory system was intact.

An immunohistochemical test for antineuronal antibodies in her serum was positive (fig 2A). Tumour markers were all negative. A gynaecological work up, radiographs of the chest, a bronchoscopical study and cytological studies of bronchial brushings and sputa disclosed no malignant lesion or cells. Gallium scintigrams showed no abnormal

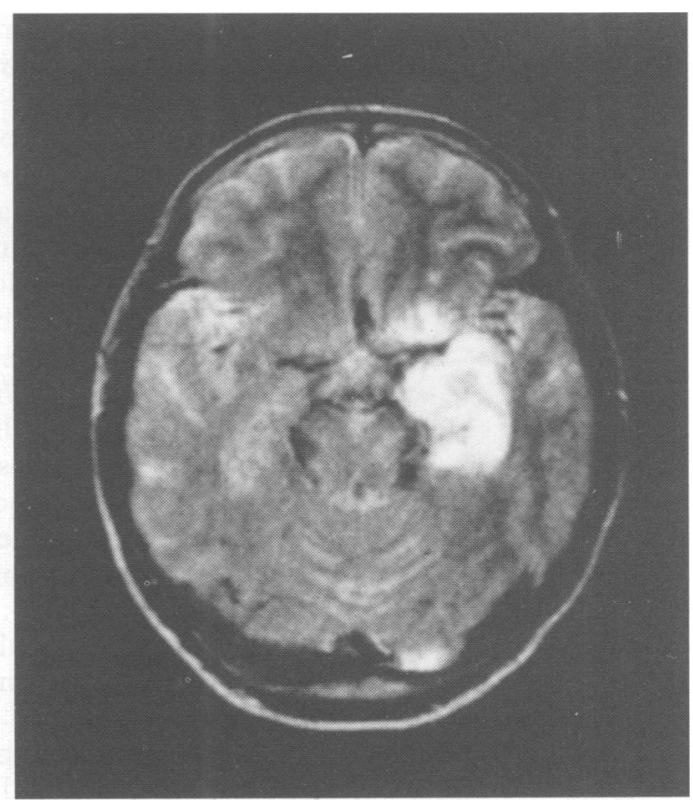

Figure $1 T 2$ weighted MRI showed a homogeneous high intensity signal with a relatively distinct margin in the medial half of the right temporal lobe and the base of the frontal lobe. The left temporal lobe also appeared to be intensified very weakly. 
Figure 2

Immunohistochemistry by the $A B C$ method. $A$ ) Cerebellum. The nuclei of Purkinje cells were strongly stained and the neuronal cytoplasma and dendrites of Purkinje cells were weakly stained. ( $\times 174)$; B) Spinal cord. The neuronal nuclei of the spinal cord were strongly, and somas weakly stained $(\times 100) ; C)$

Hippocampus. Pyramidal neurons from $C A 2$ to $C A 4$ were heavily stained, but CA1 pyramidal neurons and dentate granule cells were negative. $(\times 20)$.

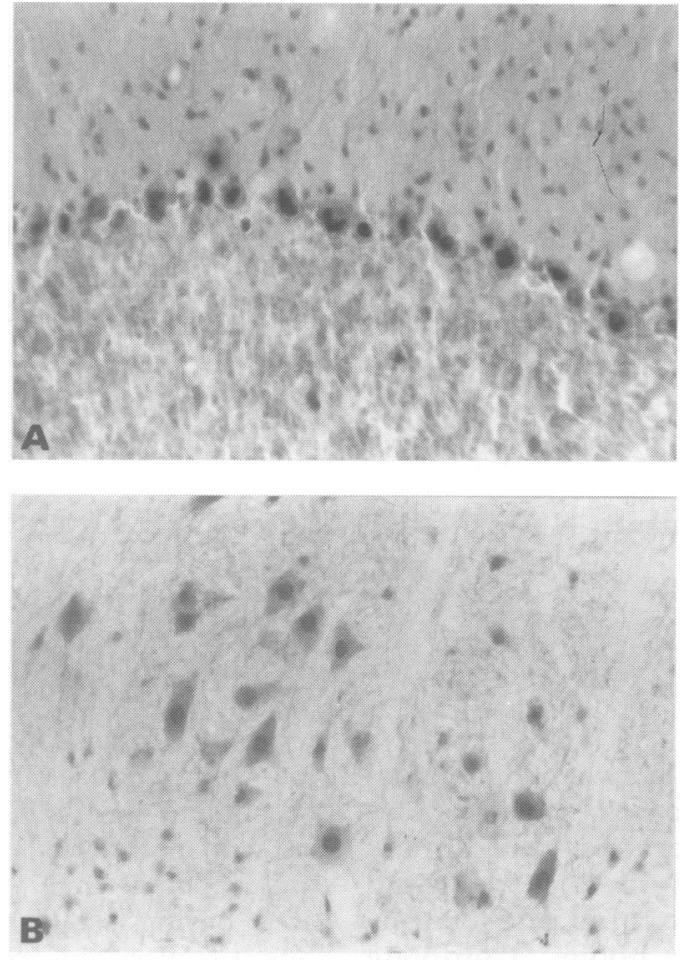

ally became generalised.

The low density area shown in the CT scan of the brain became smaller gradually. On 4 January 1989 there were no more apparent abnormal density areas and no enhancement effect by contrast medium. On 16 March, however, mild cerebral cortical atrophy and cerebellar atrophy were noted. The high intensity MRI signal also became much smaller, but was still present in the hippocampus on 6 April.

Clinically, her intellectual activity improved and no more epileptic seizures were observed. The cerebellar ataxia remained unchanged on review in May 1989.

\section{Immunohistochemistry}

Free-floating sections of $40 \mu \mathrm{m}$ thick were prepared from rat brain as previously described. ${ }^{3}$ They were incubated with serum samples diluted from $1: 2,000$ to $1: 100,000$ in PBS containing $0.3 \%$ octoxynol-9 (PBS-TX) or with undiluted CSF for six to eight days at $4^{\circ} \mathrm{C}$. After washing with PBS, the sections were incubated with anti-human biotinylated IgG diluted 1:400 in PBS-TX for two hours at room temperature. After washing with PBS, they were incubated with the avidinbiotin-horse radish peroxidase complex for two hours, and then with a diaminobenzidine solution supplemented with $1 \%$ cobalt chloride and $1 \%$ nickel ammonium sulphate. Finally they were developed with 3\% hydrogen peroxide for three to five minutes.

As a positive control, we used serum from a patient with typical paraneoplastic cerebellar degeneration ${ }^{4}$ which stained the soma and dendrites of a Purkinje cell and other neurons and recognised a $52 \mathrm{kDa}$ protein of rat brain. ${ }^{3}$

In contrast to the positive control, serum and CSF from the patient stained the nuclei of Purkinje cells, deep cerebellar nuclear neurons, spinal cord neurons and many neurons in the cerebrum and brainstem strongly and their soma and dendrites very weakly (fig $2 \mathrm{~A}$, B). Hippocampal pyramidal neurons were strongly positive from the CA2 to CA4 regions but negative in CA1. Granule cells of the dentate gyrus were also negative (fig $2 \mathrm{C}$ ). Serum diluted even up to $1: 100,000$ could adequately stain the rat brain sections, but we did not test the end point of dilution.

Two serum samples obtained from patients with spinocerebellar degeneration and three samples from normal subjects were negative.

\section{Western immunoblotting}

After cardiac perfusion with a PBS solution containing $10 \mathrm{mmol} / \mathrm{L}$ EDTA, $2 \mathrm{mmol} / \mathrm{L}$ leupeptin and $2 \mathrm{mmol} / \mathrm{L}$ pepstatin, a rat cerebellum was removed and homogenised with a glass-Teflon homogeniser in the same solution at $4^{\circ} \mathrm{C}$. The homogenate was centrifuged at $1000 \mathrm{~g}$ for 15 minutes. The supernatant was mixed with the same volume of the sample buffer $(0.125 \mathrm{M}$ Tris. $\mathrm{HCl}, \mathrm{pH} 6.8$ containing $4 \%$ SDS, $10 \%$ 2-mercaptoethanol, $20 \%$ glycerine and $0.002 \%$ bromophenol blue) for sodium dodecylsulphate gel electrophoresis (SDS-PAGE) and heated at 
Figure 3 Immunoblots of rat brain reacted with serum samples from the patient (left), a normal subject (middle) and a patient previously reported ${ }^{3}$ (right).

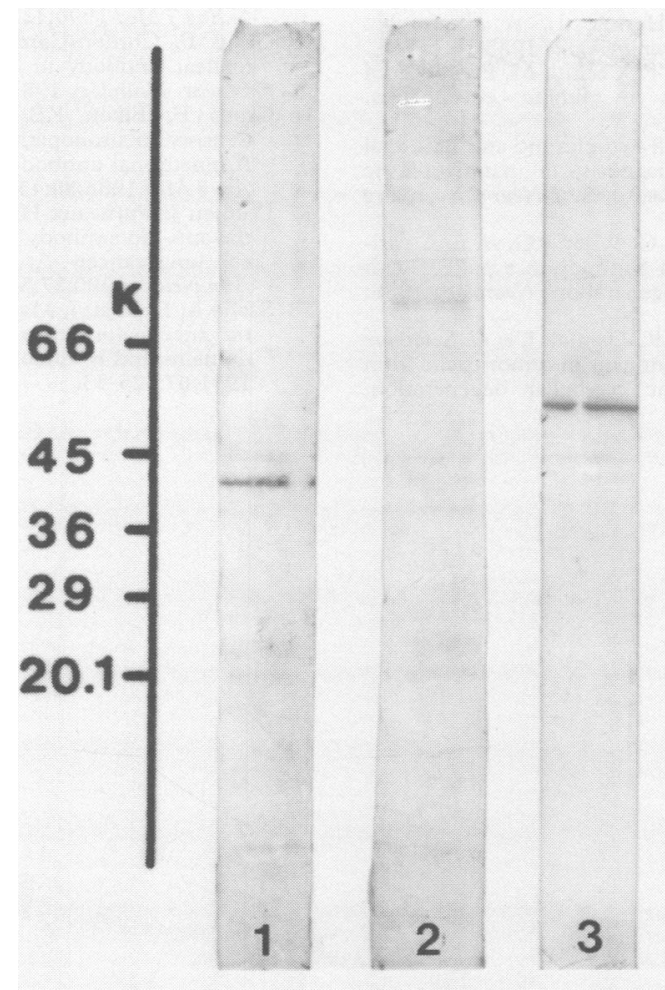

$80^{\circ} \mathrm{C}$ for 10 minutes. An aliquot of the treated sample $(10 \mu \mathrm{l}, 10 \mu \mathrm{g}$ as protein) was subjected to SDS-PAGE analysis on $10 \%$ gel. After transfer to a nitrocellulose membrane, the protein blots were immunostained with serum samples diluted 1:1000. For molecular weight estimation, we used a set of SDS molecular weight markers (MW-SDS-7OL, Sigma).

We confirmed that the positive control serum identified a $52 \mathrm{kDa}$ protein. ${ }^{3}$ In contrast, serum from the patient reacted with a distinct single protein band of $41 \mathrm{kDa}$ (fig 3, lane 1). Two serum samples from the patients with spinocerebellar degeneration and three from the normal subjects did not recognise any specific band.

\section{Discussion}

The association of paraneoplastic cerebellar degeneration and limbic encephalitis is rare, although other combinations such as paraneoplastic cerebellar degeneration and Lambert-Eaton myasthenic syndrome ${ }^{56}$ or subacute sensory neuropathy and other forms of encephalomyelitis ${ }^{7-9}$ have been reported. One malignant tumour can cause two or more different paraneoplastic disorders in a patient, but the pathological process or mechanism leading to the neurological deficits may not be the same. ${ }^{6}$

In our patient the symptoms began with slowly progressive ataxia, but during this period brain CT scans disclosed no abnormality. Later CT and MRI scans showed abnormalities consistent with limbic encephalitis ${ }^{10} 11$ which became smaller without any specific treatment. Clinically, however, epileptic seizures and EEG abnormalities became worse even after the radiological improvement. Since the temporal lobe symptoms lessened around the time of the excision of the tumour, it seems likely that the clinical improvement resulted from the removal of the tumour.

Abnormal intensity areas were not observed in the cerebellum or brainstem on CT scans or MRIs, but about 15 months after the onset of paraneoplastic cerebellar degeneration, atrophy of the cerebellum became evident.

Early diagnosis and early extirpation of the tumour are essential to curtailing the progression of neurological deficits as well as to a favourable prognosis for life. When a neurological paraneoplastic syndrome is suspected, a test for antibodies against brain tissue is an excellent diagnostic aid for confirmation of the existence of a malignant tumour. ${ }^{12-14}$

Immunohistochemical analysis showed that the patient's serum had high titres of anti-neuronal nuclear antibody against rat brain. The reaction of neurons was positive or negative and depended upon their anatomical localisations in the brain. The immunohistochemical staining pattern of this antineuronal nuclear antibody resembled that of anti-Hu antibody which often rises in sera from patients with subacute sensory neuropathy associated with small cell lung cancer. ${ }^{7815-17}$ However, Western immunoblotting gave different results. Our antibody reacted with a single protein band of $41 \mathrm{kDa}$, while anti-Hu antibody recognises several bands of 35 to 40 $\mathrm{kDa}^{781617}$; a cDNA clone that encodes a neuronal antigen $(\mathrm{HuD})$ recognised by anti$\mathrm{Hu}$ antibody has recently been isolated. ${ }^{18}$ Our patient did not show any evidence of polyneuropathy. Furthermore the causative tumour was not small cell lung cancer, but adenocarcinoma of the colon. We thus consider our antibody to be different from anti$\mathrm{Hu}$ antibody. We are now trying to clone a cDNA encoding the antigen recognised by our antibody.

1 Brain WR, Wilkinson M. Subacute cerebellar degeneration associated with neoplasma. Brain 1965;88:465-78. 2 Corsellis JAN, Goldberg GJ, Norton AR. "Limbic encephalitis" and its association with carcinoma. Brain 1968;91:481-95.

3 Tsukamoto T, Yamamoto H, Iwasaki Y, et al. Antineural autoantibodies in patients with paraneoplastic cerebellar degeneration. Arch Neurol 1989;46:1225-9.

4 Tsukamoto $T$, Yoshie O, Tada K, Iwasaki Y. AntiPurkinje cell antibody producing B-cell lines from a patient with paraneoplastic cerebellar degeneration. Arch Neurol 1987;44:833-7.

5 Satoyoshi E, Kowa H, Fukunaga N. Subacute cerebellar degeneration and Eaton-Lambert syndrome with bronchogenic carcinoma. Neurology 1973;23:764-8.

6 Blumenfeld AM, Recht LD, Chad DA, et al. Coexistence of Lambert-Eaton myasthenic syndrome and subacute cerebellar degeneration: Differential effects of treatment. Neurology 1991;41:1682-5.

7 Graus F, Elkon KB, Lloberes P, et al. Neuronal antinuclear antibody (anti-Hu) in paraneoplastic encephalonuclear antibody (anti-Hu) in paraneoplastic encephalo1987;75:249-52.

8 Anderson NE, Rosenblum MK, Graus F, et al. Autoantibodies in paraneoplastic syndromes associated with small-cell lung cancer. Neurology 1988;38:1391-8.

9 Dalmau J, Furneaux HM, Rosenblum MK, et al. Detection of the anti-Hu antibody in specific regions of the nervous system and tumor from patients with paraneoplastic encephalomyelitis/sensory neuronopathy. Neurology 1991;41:1757-64. 
10 Kohler J, Hufschmidt A, Hermle L, et al. Limbic encephalitis: two cases. $\mathcal{F}$ Neuroimmunol 1988;20:177-8.

11 Dirr LY, Elster AD, Donofrio PD, Smith M. Evolution of brain MRI abnormalities in limbic encephalitis. Neurology 1990;40:1304-6.

12 Lennon VA. Anti-Purkinje cell cytoplasmic and neuronal nuclear antibodies aid diagnosis of paraneoplastic autoimmune neurological disorders. $\mathcal{F}$ Neurol Neurosurg Psychiatry 1989;52:1438-9.

13 Anderson NE, Budde-Steffen C, Wiley RG, et al. A variant of the anti-Purkinje cell antibody in a patient with paraneoplastic cerebellar degeneration. Neurology 1988; 38:1018-26.

14 Furneaux HM, Rosenblum MK, Dalmau J, et al. Selective expression of Purkinie-cell antigens in tumor tissue from patients with paraneoplastic cerebellar degeneration.
N Eng $\mathcal{f}$ Med 1990;322:1844-51.

15 Graus F, Cordon-Cardo C, Posner JB. Neuronal antinuclear antibody in sensory neuronopathy from lung cancer. Neurology 1985;35:538-43.

16 Graus F, Elkon KB, Cordon-Cardo C, Posner JB. Sensory neuronopathy and small cell lung cancer. Antineuronal antibody that also reacts with the tumor. Antineuronal antibody that

17 Dalmau J, Furneaux HM, Gralla RJ, et al. Detection of the anti-Hu antibody in the serum of patients with small cell lung cancer-A quantitative western blot analysis. Ann Neurol 1990;27:544-52.

18 Szabo A, Dalmau J, Manley G, et al. HuD, a paraneoplastic encephalomyelitis antigen, contains RNA-binding domains and is homologous to Elav and Sex-lethal. Cell 1991;67:325-33. 